\title{
Sustained lung inflation in the delivery room in preterm infants at high risk of respiratory distress syndrome (SLI STUDY): study protocol for a randomized controlled trial
}

\author{
Carlo Dani ${ }^{1} 6^{*}$, Gianluca Lista ${ }^{2}$, Simone Pratesi ${ }^{1}$, Luca Boni ${ }^{3}$, Massimo Agosti ${ }^{4}$, Paolo Biban ${ }^{5}$, Antonio Del Vecchio ${ }^{6}$, \\ Diego Gazzolo ${ }^{7}$, Camilla Gizzi ${ }^{8}$, Rosario Magaldi ${ }^{9}$, Hubert Messner ${ }^{10}$, Fabio Mosca ${ }^{11}$, Fabrizio Sandri ${ }^{12}$, \\ Fabio Scopesi ${ }^{13}$, Daniele Trevisanuto ${ }^{14}$ and Giovanni Vento ${ }^{15}$
}

\begin{abstract}
Background: Some studies have suggested that the early sustained lung inflation (SLI) procedure is effective in decreasing the need for mechanical ventilation (MV) and improving respiratory outcome in preterm infants. We planned the present randomized controlled trial to confirm or refute these findings.

Methods/Design: In this study, 276 infants born at $25^{+0}$ to $28^{+6}$ weeks' gestation at high risk of respiratory distress syndrome (RDS) will be randomized to receive the SLI maneuver $\left(25 \mathrm{cmH}_{2} \mathrm{O}\right.$ for 15 seconds) followed by nasal continuous positive airway pressure (NCPAP) or NCPAP alone in the delivery room. SLI and NCPAP will be delivered using a neonatal mask and a T-piece ventilator.

The primary endpoint is the need for MV in the first 72 hours of life. The secondary endpoints include the need and duration of respiratory support (NCPAP, MV and surfactant), and the occurrence of bronchopulmonary dysplasia (BPD).
\end{abstract}

Trial registration: ClinicalTrials.gov identifier: NCT01440868

Keywords: Sustained lung inflation, Preterm infants, Resuscitation, Delivery room, Mechanical ventilation

\section{Background}

The pathogenesis of bronchopulmonary dysplasia (BPD) in preterm infants is multifactorial, but the role of ventilator-induced lung injury (VILI) is very important [1]. Although the respiratory support of preterm infants with respiratory distress syndrome (RDS) has improved and new modes of mechanical ventilation (MV) have been developed, the occurrence of BPD has remained substantially unchanged. Nevertheless, one aspect of respiratory care in preterm infants which has not yet been thoroughly investigated is respiratory assistance in the delivery room immediately after birth. In fact, lung protection should start in

\footnotetext{
* Correspondence: cdani@unifi.it

'Department of Surgical and Medical Critical Care, Section of Neonatology,

Careggi University Hospital, Viale Morgagni 85, 50141 Florence, Italy

${ }^{16}$ Division of Neonatology, Careggi University Hospital, University of Florence

School of Medicine, Viale Morgagni, 85, 50141 Florence, Italy

Full list of author information is available at the end of the article
}

the delivery room where, from the first breaths, the preterm infant can be helped to clear the lung fluid; to recruit alveolar spaces facilitating the formation of the functional residual capacity (FRC); to protect the lung by avoiding large tidal volume; to avoid the continuous 'opening and closing' of the alveoli by delivering positive end-expiratory pressure (PEEP); and to verify the need for surfactant replacement $[2,3]$.

Recently, some studies have investigated the effectiveness of the early sustained lung inflation (SLI) procedure in preterm infants for prevention of MV. This strategy would permit lung recruitment immediately after birth through delivery of brief peak pressure to the infant airways via a nasopharyngeal tube or mask allowing preterm infants to achieve FRC. To avoid lung collapse at the end of the expiration, the effect of PEEP is crucial. SLI and PEEP seem to have an additive effect on adequate FRC formation by 
permitting optimal gas exchange, improving lung mechanics and reducing the need for respiratory support.

This technique has been proven to be more effective than intermittent mandatory ventilation (IMV) in improving FRC in the asphyxiated term newborn [4,5]. Lindner et al. treated preterm infants by using SLI in the delivery room without a significant decrease in the MV rate or adverse effects in comparison with treatment with nasalintermittent mandatory ventilation (NIMV) noted [6]. On the other hand, te Pas et al. treated preterm infants with repeatable SLI maneuvers and found a decrease in the need for MV at 72 hours of life and BPD in comparison with treatment in the delivery room with a self-inflating bag [7]. These results are in agreement with the findings of a recent paper from the same group, which demonstrates the synergistic effect of SLI and following subsequent treatment with PEEP (delivered by nasal continuous airway pressure (NCPAP)) in achieving and maintaining a FRC improvement in an animal model [8]. Moreover, Listaet al. recently reported that SLI followed by the delivery of NCPAP is effective in reducing the need for MV and the occurrence of bronchopulmonary dysplasia in survivors compared to NCPAP alone [9].

We therefore intend to compare the application of SLI followed by NCPAP with NCPAP alone in the delivery room to evaluate its effectiveness in decreasing the need for MV and improving respiratory outcome in preterm infants at risk for RDS.

\section{Methods/Design}

Aims

The primary aim of this study is to compare the need for MV in the first 72 hours of life (excluding the transient tracheal intubation performed for surfactant administration) in infants born at $25^{+0}$ to $28^{+6}$ weeks' gestation who received the SLI maneuver in the delivery room or not.

\section{Study design}

This will be an unblinded multicenter randomized trial of SLI versus noSLI in infants born at $25^{+0}$ to $28^{+6}$ weeks' gestation.

\section{Inclusion criteria}

Inborn infants satisfying the following inclusion criteria will be eligible to participate in the study:

1. Born at $25^{+0}$ to $28^{+6}$ weeks' gestation (and)

2. High risk of RDS (and)

3. Parental consent has been obtained.

\section{Exclusion criteria}

1. Presence of major congenital malformations.

2. Fetal hydrops.
3. Inherited disorders of metabolism.

\section{Primary outcome measure}

The primary endpoint of this study will be the need for MV within the first 72 hours of life. Therefore, we will consider SLI a success if MV is not required and a failure if the infant needs MV. The need for MV is defined later under 'Criteria for starting MV'.

We have chosen the need for MV during the first 72 hours of life as our primary outcome measure to maintain similarity with the main previous trial conducted on this issue [7].

\section{Secondary outcome measures}

1. Need for conventional or high frequency modes of ventilation in the first 3 hours of life.

2. Highest $\mathrm{FiO}_{2}$ and oxygentherapy duration.

3. Duration of NCPAP, need and duration of bi-level nasal continuous positive airway pressure (BiPAP) and NIMV.

4. Need and duration of conventional MV (synchronized intermittent mechanical ventilation (SIMV), synchronized intermittent positive pressure ventilation (SIPPV), pressure support ventilation (PSV) with or without with volume guarantee (VG)) or high frequency ventilation (HFV).

5. Duration of hospitalization.

6. Highest mean airway pressure (MAP) during MV.

7. Need and number of doses of surfactant.

8. Occurrence of mild, moderate and severe bronchopulmonary dysplasia (BPD) [10].

9. Death.

10. Death or severe BPD.

\section{Other collected data}

The following data will be recorded for each infant: gestational age (GA), birth weight (BW), sex, Apgar score at 5 minutes, antenatal steroid treatment, CRIB II score [11], occurrence of pneumothorax, patent ductus arteriosus (PDA) and need of surgical closure, grade 3 to 4 intraventricular hemorrhage (IVH) [12], periventricular leukomalacia (PVL) [13], grade $>2$ retinopathy of prematurity (ROPand ROP requiring surgery [14], necrotizing enterocolitis (NEC) [15], sepsis [16], length of stay in neonatal intensive care unit (NICU) and hospital, and mortality. Patients will be discharged from the NICU to a lower level of care when they no longer need respiratory assistance other than oxygentherapy and central venous catheters.

\section{Sample size}

We hypothesize that SLI maneuver might decrease the need for MV during the first 72 hours of life from 35\% to $20 \%$. We calculated that 138 newborns must be 
enrolled in each group to detect this difference as statistically significant with $80 \%$ power at a level of 0.05 .

\section{Recruitment}

Written and oral information will, whenever possible, be offered to parents prior to the birth of their child if there is a risk that the mother will have a preterm delivery and the infant is likely to be eligible. Informed written consent will be signed by both the parents and sufficient time will be allowed for consent. Non-Italian speaking parents will only be asked for their consent if an adult interpreter is available. Trust interpreter and link worker services will be used to support involvement of participants whose first language is not Italian. A senior investigator will be available at all times to discuss concerns raised by parents or clinicians during the course of the trial.

A monthly accrual report of the study will be sent to participating centers.

\section{Randomization}

Infants at each unit will be assigned to a block (1st block: gestational age from $25^{+0}$ to $26^{+6}$ weeks; 2 nd block: gestational age from $27^{+0}$ to $28^{+6}$ weeks) and randomly assigned to a treatment group in 1:1 ratio using automatically generated sealed opaque envelopes, which will be prepared at Careggi University Hospital, Florence, Italy, and then distributed to participating centers. Block size will be concealed to investigators to ensure treatment balance between the two arms of the study.

\section{Blinding}

The study will not be blinded and the staff performing the study will also care for the infants later on. However, the decision to start MV will be made by clinicians other than the investigators involved in patient care and researchers assessing study end-points will be blinded to the nature of the study treatments.

To minimize bias, strict criteria and definitions will be maintained during the trial.

\section{SLI maneuver}

Infants in the SLI group will undergo the following approach. After oropharyngeal and nasal suctioning, pressure-controlled $\left(25 \mathrm{cmH}_{2} \mathrm{O}\right)$ inflation will be sustained for 15 seconds, using a neonatal mask and a T-piece ventilator, followed by the delivery of $5 \mathrm{cmH}_{2} \mathrm{O}$ NCPAP. Patients will be observed for the following 6 to 10 seconds to evaluate their cardio-respiratory function. If respiratory failure persists (that is, apnea and gasping) and/or the heart rate is $>60$ and $<100 \mathrm{bpm}$ despite NCPAP, the SLI maneuver (again $25 \mathrm{cmH}_{2} \mathrm{O}$ for 15 seconds) will be repeated. If the heart rate remains $>60$ and $<100 \mathrm{bpm}$ after the second SLI maneuver, the infant will be resuscitated following the current guidelines of the American Academy of Pediatrics (AAP) [17]. Moreover, if the heart rate remains $<60 \mathrm{bpm}$ the infant will be resuscitated following the current guidelines of the AAP [17], and a second SLI maneuver will be repeated when the heart rate increases to $>60$ and $<100 \mathrm{bpm}$. Infants in the control group will be treated with delivery of $5 \mathrm{cmH}_{2} \mathrm{O}$ NCPAP and will be assisted following the guidelines of the AAP [18].

All enrolled infants will be transferred to the NICU in NCPAP (PEEP at $5 \mathrm{cmH}_{2} \mathrm{O}$ ).

Neonatal care will be started with $\mathrm{FiO}_{2}$ of 0.21 to 0.40 in both the groups, in agreement with the local protocols. Respiratory support in the delivery room will be given using the same T-piece ventilator (Neopuff Infant T-Piece Resuscitator, Fisher \& Paykel, Auckland, New Zealand), which is a pressure-limited mechanical device that supplies consistent peak inspiratory pressure (PIP) and PEEP, and is capable of delivering sustained inflation [7]. To avoid pressure leakage, a neonatal mask of appropriate size which adequately covers both the mouth and nostrils of infants will be used. The flow rate will be set at 8 to 10 $1 /$ min without changes during the resuscitation.

\section{Criteria for starting MV}

In the delivery room, infants will be started on MV if they have not reached the goal of $70 \% \mathrm{SpO}_{2}$ by 5 minutes [19] and $85 \%$ by 10 minutes of life [17] with a heart rate $>100$ bpm, despite NCPAP at $5 \mathrm{cmH}_{2} \mathrm{O}$. In the NICU, infants will be started on $\mathrm{MV}$ when the $\mathrm{pH}$ is $<7.20$ with $\mathrm{PaCO}_{2}>65 \mathrm{mmHg}, \mathrm{PaO}_{2}<50 \mathrm{mmHg}$ with $\mathrm{FiO}_{2} \geq 0.50$ after surfactant treatment, or if infants have frequent episodes of apnea ( $>4$ episodes in 1 hour or $>2$ episodes requiring bag-and-mask ventilation), despite adequate NCPAP (5 to $7 \mathrm{cmH}_{2} \mathrm{O}$ ) delivery and oxygenation. $\mathrm{MV}$ will be set to maintain a $\mathrm{PaCO}_{2}$ of 55 to $65 \mathrm{mmHg}$ and 88 to 95\% $\mathrm{SpO}_{2}$.

\section{Other aspects of respiratory support}

To maintain an adequate $\mathrm{SpO}_{2}$, infants with an $\mathrm{FiO}_{2} \geq 0.40$ will be treated with surfactant $(200 \mathrm{mg} / \mathrm{kg}$ Curosurf, ChiesiPharmaceuticals, Parma, Italy) followed by the reinstitution of NCPAP as soon as vital signs are satisfactory (intubation-surfactant-extubation (INSURE) strategy). All infants who will need MV will be treated with surfactant and will then be gradually weaned from it. Additional doses of surfactant $(100 \mathrm{mg} / \mathrm{kg})$ will be given to infants, also through the INSURE strategy, at the discretion of the attending neonatologist.

Infants will be extubated, after a loading dose of caffeine citrate $(20 \mathrm{mg} / \mathrm{kg})$, when they meet all of the following criteria: $\mathrm{FiO}_{2}<0.40, \mathrm{PaCO}_{2}<65 \mathrm{mmHg}$ with a $\mathrm{pH}>7.20$, MAP $<7 \mathrm{cmH}_{2} \mathrm{O}$, hemodynamic stability and the absence of clinically significant patent ductus arteriosus. 


\section{Data collection}

All collected data will be obtained from the clinical records. They will be recorded on electronic data sheets designed for this study. Data will be entered by the local principal investigator on a web-based electronic case record form. Access to the form will be password protected and participants will be identified by trial number only.

Clinical information will be collected at the following times:

1. 1.At trial entry: eligibility, background information and randomization

2. 2.Following randomization: all data above listed in 'Primary outcome measure',

'Secondary outcome measures' and 'Other collected data' sections.

Further information will be collected on expected serious adverse events (SAEs).

\section{Statistical analysis}

The primary efficacy analysis will be conducted on an intention-to-treat basis. Clinical characteristics of infants in the SLI and control groups will be described using mean values and $\mathrm{SD}$, median value and range, or rate and percentage. Univariate statistical analysis will be performed using the Student'st-test for parametric continuous variables, the Wilcoxon rank-sum test for non-parametric continuous variables and the chi-square test for categorical variables. A $P<0.05$ will be considered statistically significant. SLI treatment and clinical characteristics which are most likely associated with the need for MV (gestational age, birth weight, antenatal steroids, CRIB score, INSURE procedure and hospital of birth) will be included in multiple logistic regression analysis to assess their independent role in predicting SLI success or failure. Effect estimates will be expressed as relative risk (RR) with profile likelihoodbased 95\% confidence limits.

An interim analysis is planned when 100 infants are enrolled (50 in each arm).

\section{Duration of study}

In this study, 272 infants will be recruited. The trial will terminate when the last recruited infant is discharged from hospital, or dies.

\section{Quality control and quality assurance procedures Compliance to protocol \\ Compliance will be defined as full adherence to protocol. Compliance with the protocol will be ensured by a num- ber of procedures as described below.}

\section{Site set-up}

Local principal investigators participated in preparatory meetings in which details of the study protocol, data collectionand SLI procedure were accurately discussed. All centers received detailed written instruction on web-based recording data and, to resolve possible difficulties, it will be possible to contact the Clinical Trials Coordinating Center (LB). Moreover, it has been ascertained that the SLI procedure is equally made in all participating centers, to avoid expensive visits for training at each site due to the limited resources available for this study.

\section{Data processing and monitoring}

All study data will be:

1. Screened for out-of-range data, with cross-checks for conflicting data within and between data collection forms by a data manager.

2. Referred back to the relevant center for clarification in the event of missing items or uncertainty.

3. Reviewed by the Chief Investigators (CD, GL) and trial statistician, and they will review the results generated for logic,patterns andproblems Outlier data will be investigated.

\section{Safety}

Safety end-points will include incidence, severity and causality of reported SAEs, namely changes in occurrence of the expected common prematurity complications and clinical laboratory test assessments, and the development of unexpected SAEs in this high risk population. All SAEs will be followed until satisfactory resolution or until the investigator responsible for the care of the participant deems the event to be chronic or the patient to be stable.

All expected and unexpected SAEs, whether or not they are attributable to the study intervention, will be reviewed by the local principal investigators to determine if there is a reasonable suspected causal relationship with the intervention. If the relationship is reasonable, SAEs will be reported to the Chief Investigators who will then report them to the Ethics Committee and inform all other investigators to guarantee the safety of the participants.

\section{Trial status}

The Local Ethics Committee approved the study and the trial is currently recruiting study subjects.

\footnotetext{
Abbreviations

AAP: American Academy of Pediatrics; BDP: bronchopulmonary dysplasia; BiPAP: bi-level nasal continuous positive airway pressure; BPD: bronchopulmonary dysplasia; BW: birth weight; FRC: functional residual capacity; GA: gestational age; HFV: high frequency ventilation; IMV: intermittent mandatory ventilation; INSURE: intubation-surfactantextubation; IVH: intraventricular hemorrhage; MAP: mean airway pressure; MV: mechanical ventilation; NCPAP: nasal continuous positive airway pressure; NEC: necrotizing enterocolitis; NICU: neonatal intensive care unit;
} 
NIMV: nasal intermittent mandatory ventilation; PDA: patent ductus arteriosus; PEEP: positive end-expiratory pressure; PIP: peak inspiratory pressure; PSV: pressure support ventilation; PVL: periventricular leukomalacia; RDS: respiratory distress syndrome; ROP: retinopathy of prematurity: RR: relative risk; SAE: serious adverse event; SIMV: synchronized intermittent mechanical ventilation; SIPPV: synchronized intermittent positive pressure ventilation; SLI: sustained lung inflation; VG: volume guarantee; VILI: ventilator-induced lung injury.

\section{Competing interests}

The authors declare that they have no competing interests.

\section{Authors' contributions}

$C D, G L, S P, M A, P B, A D V, D G, C G, R M, H M, F M, F S, F S, D T$ and GV have made substantial contributions to the conception and design of the study protocol, and have given final approval of the version to be published. LB prepared electronic data sheets, wasresponsible for the web-based electronic case record form and has given final approval of the version to be published. All authors read and approved the final manuscript.

\section{Acknowledgments}

We thank the following for partially funding this trial: Chiesi (Parma, Italy), Fisher \& Paykel, (Auckland, New Zealand) and Drager (Lubeck, Germany). Their generosity will permit payment of thestudy insurance (mandatory in Italy), fund all investigators' meetings, and to project, realize and manage the web-based electronic case record form. Moreover, Fisher \& Paykel will provide Neopuff Infant T-Piece Resuscitator to some centers lacking the device.

\section{Author details}

'Department of Surgical and Medical Critical Care, Section of Neonatology, Careggi University Hospital, Viale Morgagni 85, 50141 Florence, Italy. ${ }^{2}$ Division of Neonatology, 'VBuzzi' Children's Hospital, Via Castelvetro. 32, 20154 Milan, Italy. ${ }^{3}$ Clinical Trials Coordinating Center, IstitutoToscanoTumori, Florence; Department of Human Pathology and Oncology, University of Florence, Viale Morgagni 85, 50141 Florence, Italy. ${ }^{4}$ Maternal and Child Health Department, Del Ponte Hospital, A.O. Di Circolo Fondazione Macchi, Via Del Ponte, 19, 21100 Varese, Italy. ${ }^{5}$ Department of Pediatrics, Pediatric and Neonatal intensive Care Unit, Azienda Ospedaliera Universitaria Integrata, Oiazzale Stefani, 1, 37126 Verona, Italy. ${ }^{6}$ Division of Neonatology, Neonatal Intensive Care Unit, Di Venere Hospital, Bari, Via Ospedale di Venere 150, 70012, Bari, Italy. ${ }^{7}$ Department of Maternal Fetal and Neonatal Medicine, C. Arrigo Children's Hospital, Via Santa Caterina da Siena, 30, 15121 Alessandria, Italy. ${ }^{8}$ Division of Neonatology, S. Giovanni Calibita Hospital Fatebenefratelli, Piazza Fatebenefratelli 1, 00186 Rome, Italy. ${ }^{9}$ Division of Neonatology, Neonatal Intensive Care Unit, Ospedali Riuniti, Via degli Aviatori, 1, 71122 Foggia, Italy. ${ }^{10}$ Neonatal Intensive Care Unit, Ospedale Regionale, Via L. Böhler, 5, 39100 Bolzano, Italy. ${ }^{11}$ Department of Mother and Infant Science, Neonatal Intensive Care Unit, Fondazione IRCCS 'Ca' Granda'Ospedale Maggiore Policlinico, Via Commenda, 12, 20122 Milan, Italy. ${ }^{12}$ Maternal and Pediatrics Department, Maggiore Hospital, Largo Nigrisoli, 2, 40100 Bologna, Italy. ${ }^{13}$ Department of Neonatology Obstetrics and Neuroscience, G. Gaslini Children's University Hospital, Via Gerolamo Gaslini, 5, 16148 Genoa, Italy. ${ }^{14}$ Department of Pediatrics, Azienda Ospedaliera of Padua, Viale Giustiniani, 2, 35100 Padua, Italy. ${ }^{15}$ Division of Neonatology, Catholic University of Rome, Largo Agostino Gemelli 8, 00168 Rome, Italy. ${ }^{16}$ Division of Neonatology, Careggi University Hospital, University of Florence School of Medicine, Viale Morgagni, 85, 50141 Florence, Italy.

Received: 16 July 2012 Accepted: 19 February 2013

Published: 8 March 2013

\section{References}

1. Van Marter $\amalg$, Allred EN, Pagano M, Sanocka U, Parad R, Moore M, Susser M, Paneth N, Leviton A: Do clinical markers of barotrauma and oxygen toxicity explain interhospital variation in rates of chronic lung disease? The Neonatology Committee for the Developmental Network. Pediatrics 2000, 105:1194-1201.

2. Lindner W, Vossbeck S, Hummler H, Pohlandt F: Delivery room management of extremely low birth weight infants: spontaneous breathing or intubation? Pediatrics 1999, 103:961-967.
3. te Pas AB, Siew M, Wallace MJ, Kitchen MJ, Fouras A, Lewis RA, Yagi N, Uesugi K, Donath S, Davis PG, Morley CJ, Hooper SB: Effect of sustained inflation length on establishing functional residual capacity at birth in ventilated premature rabbits. Pediatr Res 2009, 66:295-300.

4. Boon AW, Milner AD, Hopkin IE: Lung expansion, tidal exchange, and formation of the functional residual capacity during resuscitation of asphyxiated neonates. JPediatr 1979, 95:1031-1036.

5. Vyas H, Milner AD, Hopkin IE, Boon AW: Physiologic responses to prolonged and slow-rise inflation in the resuscitation of the asphyxiated newborn infant. JPediatr 1981, 99:635-639.

6. Lindner W, Högel J, Pohlandt F: Sustained pressure-controlled inflation or intermittent mandatory ventilation in preterm infants in the delivery room? A randomized, controlled trial on initial respiratory support via nasopharyngeal tube. Acta Paediatr 2005, 94:303-309.

7. te $P a s A B$, Walther $F J$ : A randomized, controlled trial of delivery-room respiratory management in very preterm infants. Pediatrics 2007, 120:322-329.

8. te Pas AB, Siew M, Wallace MJ, Kitchen MJ, Fouras A, Lewis RA, Yagi N, Uesugi K, Donath S, Davis PG, Morley CJ, Hooper SB: Establishing functional residual capacity at birth: the effect of sustained inflation and positive end expiratory pressure in a preterm rabbit model. Pediatr Res 2009, 65:537-541.

9. Lista G, Fontana P, Castoldi F, Cavigioli F, Dani C: Does sustained lung inflation at birth improve outcome of preterm infants at risk for respiratory distress syndrome? Neonatology 2011, 99:45-50.

10. Jobe A, Bancalari E: Bronchopulmonarydysplasia. Am J RespirCrit Care Med 2001, 163:1723-1729.

11. Parry G, Tucker J, Tarnow-Mordi W, for the UK Neonatal Staffing Study Collaborative Group: CRIB II: an update of the clinical risk index for babies score. Lancet 2003, 361:1789-1791.

12. Papile LS, Burstein J, Burstein R, Keffler H: Incidence and evolution of the sub-ependymal intraventricular hemorrhage; a study of infants weighing less than 1500 grams. JPediatr 1978, 92:529-534.

13. De Vries LS, Eken P, Dubowitz LM: The spectrum of leukomalacia using cranial ultrasounds. Behav Brain Res 1992, 49:1-6.

14. International Committee for the Classification of Retinopathy of Prematurity: The International Classification of Retinopathy of Prematurity revisited. ArchOphthalmol 2005, 123:991-999.

15. Bell MJ, Ternberg JL, Feigin RD, Keating JP, Marshall R, Barton L, Brotherton T: Neonatal necrotizing enterocolitis: Therapeutic decisions based upon clinical staging. AnnSurg 1978, 187:1-12.

16. American Academy of Pediatrics: Committee on drugs, Committee on fetus and newborn and Committee on Infectious Diseases. Pediatrics 1980, 65:1047-1053.

17. Vento $M$, Cheung PY, Aguar M: The first golden minutes of the extremelylow-gestational-age neonate: a gentle approach. Neonatology 2009, 95:286-298.

18. Perlman JM, Wyllie J, Kattwinkel J, Atkins DL, Chameides L, Goldsmith JP, Guinsburg R, Hazinski MF, Morley C, Richmond S, Simon WM, Singhal N, Szyld E, Tamura M, Velaphi S, Neonatal Resuscitation Chapter Collaborators: International Consensus on Cardiopulmonary Resuscitation and Emergency Cardiovascular Care Science with Treatment Recommendations. Pediatrics 2010, 2010(126):e1319-e1344.

19. Finer $\mathrm{N}$, Leone $\mathrm{T}$ : Oxygen saturation monitoring for the preterm infant: the evidence basis for current practice. Pediatr Res 2009, 65:375-380.

\section{doi:10.1186/1745-6215-14-67}

Cite this article as: Dani et al:: Sustained lung inflation in the delivery room in preterm infants at high risk of respiratory distress syndrome (SLI STUDY): study protocol for a randomized controlled trial. Trials 2013 14:67. 